\title{
A role for GATA3 in ulcerative colitis
}

A new study has confirmed the role of the transcription factor GATA3 in ulcerative colitis, demonstrating that inhibition of GATA3 reduces colitis and cytokine levels in experimental models. The findings highlight the potential of therapeutic targeting of transcription factors that control cytokine production in IBD.

"The transcription factors controlling T-cell differentiation in ulcerative colitis are poorly characterized," explains author Markus Neurath. "We assumed that GATA3 as a key regulator of T-helper $\left(\mathrm{T}_{\mathrm{H}}\right)$-2 differentiation might be involved [in disease development]." As DNAzymes can be used to suppress transcription factors, Neurath and colleagues decided to adopt this approach to investigate the therapeutic potential of a GATA3-targeting DNAzyme in experimental settings.

First, GATA3 levels were found to be elevated in colon tissue samples from patients with ulcerative colitis, but not in ileal Crohn's disease or control tissue from healthy individuals. Levels of GATA3 mRNA correlated with levels of inflammatory cytokines (such as IL-6 and IL-9) and in particular to cytokine expression of $\mathrm{T}_{\mathrm{H}} 2$ and $\mathrm{T}_{\mathrm{H}} 9$ cells. Next, similar findings were observed in mice, with increased expression of GATA3 by lamina propria $\mathrm{T}$ cells in mice with oxazolone-induced colitis, compared with controls. Importantly, mice with T-cell-specific deletion of GATA3 did not develop colitis
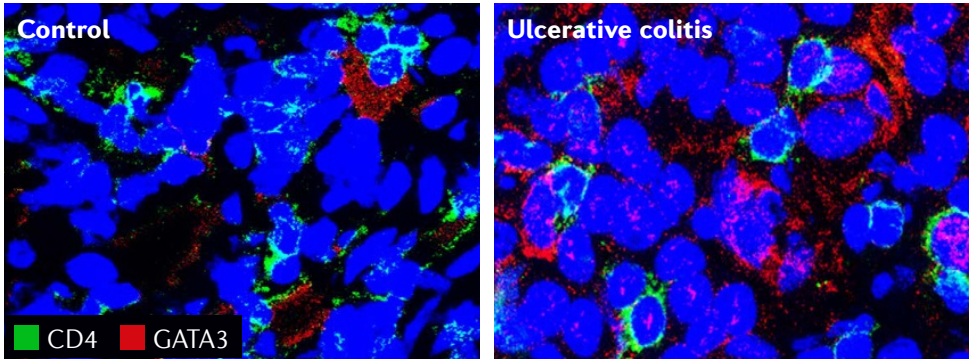

$\mathrm{CD} 4^{+} \mathrm{GATA} 3^{+} \mathrm{T}$ cells in the colon tissue of a healthy individual as control and a patient with colitis. Image courtesy of B. Weigmann. and the production of inflammatory cytokines (including IL-6, IL-9 and IL-13) was markedly reduced.

Finally, the investigators tested the GATA3 DNAzyme hgd40 in experimental colitis. In vitro studies confirmed that hgd40 suppressed GATA3 mRNA expression in T cells. Examination of the uptake and distribution of hgd40 after rectal administration in mice revealed distribution throughout the inflamed colon, with uptake within $1 \mathrm{~h}$ and localization to the mucosa after $2 \mathrm{~h}$. Importantly, intrarectal administration of hgd40 suppressed colitis in mice in two different experimental models, with substantial suppression of endoscopic signs of colitis. Hgd40 had comparable antiinflammatory effects to an anti-TNF agent in mice with colitis, and seemed to suppress cytokine production in a TNF-independent manner.

"The findings suggest that GATA3 is an important regulator of proinflammatory cytokine production in colitis and therefore an interesting new target for clinical therapy," notes Neurath. As a GATA3 DNAzyme has been shown to be beneficial as a therapy for patients with asthma, the researchers plan to set up clinical studies for this approach in patients with IBD.

Katrina Ray

ORIGINAL ARTICLE Popp, V. et al. Rectal delivery of a DNAzyme that specifically blocks the transcription factor GATA3 reduces colitis in mice. Gastroenterology http://dx.doi.org/10.1053/ j.gastro.2016.09.005 (2016) 